\title{
Burnout em Profissionais da Saúde Portugueses: Uma Análise a Nível Nacional
}

\author{
Burnout in Portuguese Healthcare Professionals: \\ An Analysis at the National Level
}

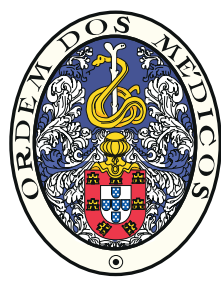

\author{
João MARÔCOه® ${ }^{1}$, Ana Lúcia MARÔCO ${ }^{2}$, Ema LEITE ${ }^{3,4}$, Cristina BASTOS ${ }^{5}$, Maria José VAZÃO ${ }^{6}$, Juliana CAMPOS $^{7}$ \\ Acta Med Port 2016 Jan;29(1):24-30
}

RESUMO

Introdução: O burnout é uma síndrome psicológica, caracterizada por elevada exaustão emocional, elevada despersonalização e baixa realização profissional, que conduz à erosão dos valores pessoais, profissionais e de saúde. Este estudo reporta a prevalência do burnout em profissionais de saúde Portugueses.

Material e Métodos: Os níveis de burnout foram estimados pelo Maslach Burnout Inventory - Human Services Survey numa escala ordinal de zero (nunca) a seis (sempre) pontos. A amostra foi constituída por 1262 enfermeiros e 466 médicos com médias de idade de 36,8 anos $(\mathrm{DP}=12,2)$ e $38,7(\mathrm{DP}=11,0)$, respetivamente. Os participantes foram provenientes de todos os distritos nacionais $(35 \%$ Lisboa; $18 \%$ Porto; $6 \%$ Aveiro, 6\% Setúbal, 5\% Coimbra; 5\% regiões autónomas), com atuação em meio hospitalar (54\%), centros de saúde (Unidade de Saúde Familiar - 30\%; Unidades de Cuidados de Saúde Primários - 8\%) e outras instituições públicas/privadas (8\%).

Resultados: A análise dos níveis de burnout revelou que ambas as categorias profissionais apresentaram níveis moderados a elevados de burnout $(M=3,0 ; D P=1,7)$ não sendo significativas as diferenças entre as duas profissões. Vila Real $(M=3,8 ; S D=$ $1,7)$ e a Madeira $(M=2,5 ; D P=1,5)$ são as regiões onde os níveis de burnout são mais e menos elevados, respetivamente. Os níveis de burnout não diferiram significativamente entre Hospitais, Unidades de Cuidados de Saúde Personalizados e Unidades de Saúde Familiares. Os profissionais com maior tempo na função são menos acometidos por burnout $(r=-0,15)$ não ocorrendo associação significativa com a duração da jornada de trabalho $(r=0,04)$. A má qualidade das condições de trabalho foi o melhor preditor do burnout $(r=-0,35)$.

Discussão: A ocorrência da síndrome de burnout em profissionais de saúde portugueses é frequente, estando associada à percepção de más condições de trabalho e à menor duração do tempo de serviço. A incidência de burnout apresenta diferenças regionais que podem estar associadas ao aumento do stress imposto pelo exercício da profissão em condições sub-ótimas para a prestação dos cuidados de saúde. Os resultados alertam para a necessidade de intervenções para melhorar as condições de trabalho e formação inicial dos profissionais de saúde de forma a garantir a qualidade do serviço prestado aos utentes e o bem-estar pessoal destes profissionais. Conclusões: A nível nacional, entre 2011 e 2013, 21,6\% dos profissionais de saúde apresentaram burnout moderado e 47,8\% burnout elevado. A perceção de más condições de trabalho foi o principal preditor da ocorrência de burnout nos profissionais de saúde Portugueses.

Palavras-chave: Esgotamento Profissional; Pessoal de Saúde; Portugal.

\section{ABSTRACT}

Introduction: Burnout is a psychological syndrome, characterized by a state of high emotional exhaustion, high depersonalization and low personal accomplishment, which leads to erosion of personal, professional and health values. This study reports the incidence of burnout in Portuguese Healthcare professionals.

Material and Methods: Burnout in Portugal's health professionals was assessed with the Maslach Burnout Inventory - Human Services Survey, using a zero (never) to six (always) ordinal scale, on a sample of 1262 nurses and 466 physicians with mean age of 36.8 year $(\mathrm{SD}=12.2)$ and $38.7(\mathrm{SD}=11.0)$, respectively. Participants came from all national districts $(35 \%$ Lisbon, $18 \%$ Oporto, $6 \%$ Aveiro, $6 \%$ Setúbal, $5 \%$ Coimbra, $5 \%$ Azores and Madeira), working in hospitals (54\%), Families' Health Units (30\%), Personalized Health Units $(8 \%)$ and other public or private institutions (8\%).

Results: Analysis of MBI-HSS scores, stratified by district, revealed that both types of professionals had moderate to high levels of burnout $(M=3.0, S D=1.7)$ with no significant differences between the two groups. Vila Real $(M=3.8, S D=1.7)$ and $M a d e i r a(M=2.5$, $S D=1.5$ ) were the regions where burnout levels were higher and lower, respectively. Burnout levels did not differ significantly between Hospital, Personalized Health Units and Families' Health Centers. Professionals with more years in the function were less affected by Burnout $(r=-0.15)$. No significant association was observed with the duration of the working day $(r=0.04)$. The strongest predictor of burnout was the perceived quality of working conditions $(r=-0.35)$.

Discussion: The occurrence of the burnout syndrome in Portuguese health professionals is frequent, being associated with a poor working conditions perception and reduced professional experience. The incidence of the burnout syndrome shows regional differences which may be associated with different and suboptimal conditions for health care delivery. Results suggest the need for interventions

1. Departamento de Ciências Psicológicas. William James Center for Research. Instituto Superior de Psicologia Aplicada. Lisboa. Portugal.

2. Seç̧ão de Estatística. Escola Superior de Comunicação Social. Lisboa. Portugal.

3. Grupo de Disciplinas de Saúde Ocupacional. Escola Nacional de Saúde Pública. Lisboa. Portugal.

4. Serviço de Saúde Ocupacional. Hospital de Santa Maria. Centro Hospitalar Lisboa Norte. Lisboa. Portugal.

5. Serviço de Medicina Geral e Familiar. Agrupamento de Centros de Saúde Lisboa Ocidental e Oeiras. Oeiras. Portugal.

6. Departamento de Enfermagem. Agrupamento de Centros de Saúde Lisboa Ocidental e Oeiras. Oeiras. Portugal.

7. Departamento de Odontologia. Faculdade de Odontologia de Araraquara. Universidade Estatual de São Paulo. São Paulo. Brasil.

$\triangle$ Autor correspondente: João Marôco. joao.maroco@ispa.pt

Recebido: 28 de março de 2015 - Aceite: 26 de agosto de 2015 | Copyright $\odot$ Ordem dos Médicos 2016 
aimed at improving working conditions and initial job training of health professionals, as requisites for a good professional practice and personal well-being.

Conclusions: At the national level, between 2011 and 2013, 21.6\% of healthcare professionals showed moderate burnout and $47.8 \%$ showed high burnout. The perception of poor working conditions was the main predictor of burnout occurrence among the Portuguese health professionals.

Keywords: Burnout, Professional; Health Personnel; Portugal.

\section{INTRODUÇÃO}

O termo burn-out ou burnout é proveniente do inglês e significa 'queimar até à exaustão'. O termo foi proposto pela primeira vez pelo professor e psicanalista americano, de origem alemã, Herbert Freudenberger ${ }^{1}$ depois de o constatar em si mesmo e em colegas a trabalhar no free-clinic movement. Segundo a definição de Freundenberg, o burnout é um "state of mental and physical exhaustion caused by one's professional life" que indica um conjunto de sinais e sintomas associados ao colapso físico e emocional que sobrevêm após a exaustão de toda energia, recursos ou forças disponíveis na realização de tarefas de 'ajuda' a outros. Apesar da existência de vários referenciais teóricos, no contexto da Psicologia o referencial mais utilizado é o de Maslach e Jackson. ${ }^{2,3}$ Segundo estes autores, o burnout é uma síndrome multifatorial constituída por exaustão emocional, desumanização/despersonalização e reduzida realização no trabalho que pode observar-se em indivíduos com profissões onde existe interação/ajuda com ou a outras pessoas. Ferreira e Lucca ${ }^{4}$ afirmam que esta síndrome é reflexo de um processo contínuo de sentimentos de inadequação em relação ao trabalho e à falta de recursos para realizar esse trabalho. O burnout, se não for devidamente intervencionado, pode causar um forte desgaste físico e emocional e associar-se a sintomatologia física como cefaleias, tonturas, dispneia, distúrbios de sono. Associa-se igualmente a alterações psicológicas como labilidade emocional, irritabilidade, ira e ansiedade e ainda a dificuldade de relacionamento social. A baixa produtividade e os conflitos de natureza laboral, a dependência de substâncias psicotrópicas, a redução da satisfação com o trabalho e com a vida pessoal, numa espiral de sofrimento, pode conduzir, em casos mais graves, ao suicídio.,

Gil-Monte $^{7}$ considera que a síndrome de burnout é um problema de saúde pública considerando as suas implicações na saúde física e mental do trabalhador, com o compromisso da sua qualidade de vida. Num estudo de 2011, publicitado pela Academia Americana de Cirurgiões Ortopédicos, foi observado que $87 \%$ de mais de 2000 médicos dos Estados Unidos reportaram sentir-se severamente stressados e em burnout num dia regular de trabalho. ${ }^{8} \mathrm{O}$ burnout tem sido, por isso, considerado um problema de saúde ocupacional de grande relevância entre a classe dos profissionais de saúde. Indivíduos com burnout, geralmente, apresentam uma redução da qualidade do desempenho profissional, com maior probabilidade de erro médico, maiores taxas de absentismo, menor compromisso com a função e com o empregador, diminuição da satisfação no trabalho, maior ocorrência de baixas médicas, maior sofrimento pessoal e aumento dos conflitos interpessoais envolvendo chefias, colegas e família, abuso de álcool e outros psicotrópicos e menores níveis de exercício físico ou de outras atividades de vida saudáveis. ${ }^{3,9,10}$

Os primeiros trabalhos sobre burnout referiam-se exclusivamente a profissões do tipo assistencialista, como por exemplo assistentes sociais, enfermeiros e psicólogos. ${ }^{11}$ Atualmente, sabe-se que o burnout atinge um contexto mais alargado de profissionais de profissões de ajuda (professores, policias, bombeiros, $\ldots)^{12-14}$ bem como outras atividades que não sendo profissões remuneradas apresentam algumas características comuns às profissões remuneradas (e.g., estudantes em contexto universitário). ${ }^{15,16}$ Ferreira e Lucca ${ }^{4}$ reforçam que os profissionais de saúde são especialmente vulneráveis ao desenvolvimento da síndrome de burnout devido ao contacto quotidiano com pessoas debilitadas/doentes além de, muitas vezes, terem que lidar com relações interpessoais tensas e hierárquicas nas instituições de saúde. Outro aspeto, destacado por estes autores, é a estrutura do horário de trabalho (turnos com trabalho noturno) que podem contribuir para a sobrecarga física, cognitiva e emocional dos profissionais de saúde.

Entre os profissionais de saúde, destacam-se a vulnerabilidade de enfermeiros e médicos ao desenvolvimento da síndrome de burnout. Apesar do diagnóstico de níveis elevados de burnout em profissionais de saúde não ser recente, o tema é cada vez mais atual a avaliar pela quantidade de estudos sobre a síndrome de burnout, as suas causas, efeitos e estratégias de prevenção nestas categorias profissionais, publicados nos últimos anos. ${ }^{17-26}$ Por exemplo, em Portugal, um estudo recente realizado por Queirós et al ${ }^{25} \mathrm{em}$ quatro hospitais da cidade do Porto, procurou identificar os preditores do burnout em enfermeiros tendo verificado que o género, a idade, os anos de experiência na função, satisfação no trabalho e a interação trabalho/casa foram preditores significativos da incidência de burnout nestes profissionais. Contudo, com a exceção do estudo de Marcelino et a ${ }^{27}$ numa amostra de 153 médicos de família distribuídos por todo o território nacional, onde foi usada uma versão do instrumento de medida não previamente validada, não temos conhecimento de outros estudos publicados que tenham avaliado a incidência de burnout e os seus preditores em médicos e enfermeiros, a nível nacional.

Em nosso entender, é necessária uma avaliação generalizada, e representativa, da incidência do burnout, de forma a sustentar intervenções profissionais e políticas públicas de maior abrangência na promoção da saúde ocupacional. Assim, este estudo teve como objetivos: (1) fazer a avaliação dos níveis de burnout em médicos e enfermeiros em diferentes contextos profissionais, em todos os distritos e regiões autónomas do país e (2) avaliar a sua associação com variáveis sociodemográficas e laborais. 
Tabela 1 - Caracterização sociodemográfica dos participantes. Portugal, 2011-2013.

\begin{tabular}{lcc}
\hline Variável & \multicolumn{2}{c}{ Profissão } \\
\hline $\mathrm{n}(\%)$ & Enfermeiro(a) & Médico(a) \\
Género (\% M/F) & $1262(73)$ & $466(27)$ \\
Local trabalho (\% Hospital/USF/Outro) & $17 / 83$ & $42 / 58$ \\
Idade (anos; média \pm desvio-padrão & $(63 / 36 / 1)$ & $(84 / 12 / 4)$ \\
Anos na função (média \pm desvio-padrão) & $39 \pm 12$ & $37 \pm 9$ \\
Horas trabalho/semana (média \pm desvio-padrão) & $12 \pm 9$ & $11 \pm 8$ \\
\hline
\end{tabular}

\section{MATERIAL E MÉTODOS \\ Participantes}

Foram convidados a participar no estudo médicos e enfermeiros de Portugal continental e regiões autónomas dos Açores e Madeira, de ambos os sexos, recrutados por convite em serviços de medicina ocupacional e por divulgação do estudo em associações socioprofissionais (Ordem dos Enfermeiros, Associação dos Médicos de Família e Clínica Geral, Sindicato dos Médicos do Norte).

As características sociodemográficas dos 1728 profissionais que concordaram em participar no estudo são apresentadas na Tabela 1

O contexto profissional dos participantes ocorre com maior frequência em meio hospitalar e em Unidades de Saúde Familiares.

Do total de participantes neste estudo, apenas 1685 indicaram o distrito/região autónoma onde exerciam a sua atividade profissional (Tabela 2).

Os participantes são provenientes de todos os distritos nacionais, mas com maior expressão em Lisboa (34,5\%); Porto $(18,1 \%)$, Setúbal $(6,1 \%)$, Aveiro $(6,0 \%)$, Coimbra $(5,6 \%)$ e Regiões autónomas (5\%).

\section{Instrumentos de Medida}

O Maslach Burnout Inventory - Human Services Survey $(\mathrm{MBI}-\mathrm{HSS})^{28}$ é o instrumento de avaliação de burnout usado em mais de $95 \%$ dos estudos sobre burnout. ${ }^{29} \mathrm{~A}$ versão do MBI-HSS usada neste estudo foi traduzida e adaptada transculturalmente para Portugal e Brasil pelos autores após autorização do detentor do copyright do instrumento (MindGarden $\left.{ }^{\oplus} 1986\right)$. A versão portuguesa do MBI-HSS é constituída por 22 itens ordinais que refletem as três dimensões do burnout: exaustão emocional (composta por nove itens p. ex. 'Item 1: O meu trabalho deixa-me emocionalmente esgotado'); descrença/despersonalização (composta por cinco itens p. ex. 'Item 5: Sinto que trato alguns dos meus clientes de forma impessoal') e Realização profissional (composta por oito itens p. ex. 'Item 9: Sinto que influencio positivamente a vida das pessoas através do meu trabalho'). O formato de resposta é ordinal com sete pontos de ' 0 - Nunca/Nenhuma vez' a ' 6 - Sempre/Todos os dias'. Face à ausência de validade fatorial da versão original da MBI-HSS na amostra sob estudo $\left(\chi^{2} / g \mid=2,4\right.$; $\mathrm{CFI}=0,781 ; \mathrm{GFI}=0,704 ; \mathrm{TLI}=0,755, \mathrm{RMSEA}=0,114)$, foi
Tabela 2 - Distribuição percentual dos $\mathrm{n}=1685$ participantes que indicaram o distrito/região autónoma onde exerciam sua atividade profissional. Portugal, 2011-2013.

\begin{tabular}{lc}
\hline Distrito/Região & $\mathbf{( \% )}$ \\
\hline Aveiro & 6,0 \\
Beja & 1,4 \\
Braga & 3,5 \\
Bragança & 1,4 \\
Castelo Branco & 1,0 \\
Coimbra & 5,6 \\
Évora & 0,6 \\
Faro & 3,1 \\
Guarda & 0,6 \\
Leiria & 2,2 \\
Lisboa & 34,5 \\
Portalegre & 0,4 \\
Porto & 8,1 \\
Região Autónoma dos Açores & 2,6 \\
Região Autónoma da Madeira & 2,4 \\
Santarém & 2,4 \\
Setúbal & 3,1 \\
Viana do Castelo & 6,1 \\
Vila Real & 1,6 \\
\hline & 2,1 \\
\hline
\end{tabular}

produzida uma versão reduzida com cinco itens por dimensão (exaustão emocional: itens 1,3,8,14,20; despersonalização: itens $5,10,11,15,22$ e realização profissional: itens $9,17,18,19,21)$ com boa validade fatorial $\left(\alpha^{2} / \mathrm{gl}=3,6\right.$; $\mathrm{CFI}=$ 0,$957 ; \mathrm{GFI}=0,956 ; \mathrm{TLI}=0,948, \mathrm{RMSEA}=0,027$ ) e elevada fiabilidade $\left(\alpha_{\text {Exaustäo }}=0,87 ; \alpha_{\text {Descrença }}=0,72 ; \alpha_{\text {Realização }}=0,82\right)$. O score global de burnout foi calculado, usando os factor score weights obtidos num modelo fatorial onde o burnout é definido como um constructo de segunda ordem que se manifesta nas dimensões de primeira ordem exaustão 
( $\beta=0,73, p<0,001)$, despersonalização $(\beta=0,76, p<$ $0,001)$ e realização profissional $(\beta=-0,69, p<0,001)$. 0 score global de burnout foi depois convertido em classes de burnout seguindo a recomendação de Maslach et al (1986): 'Sem burnout/burnout reduzido' para scores médios inferiores a dois; 'burnout moderado' para scores médios entre [2; 3[ e 'burnout elevado' para scores médios superiores ou iguais a três.

As características sociodemográficas dos participantes, percursos profissionais e locais de trabalho foram registadas com um questionário sociodemográfico.

\section{Procedimentos}

Os participantes foram informados que a sua participação era voluntária e anónima; que poderiam desistir em qualquer momento; que o estudo apresentava objetivos puramente científicos sem intervenção clínica ou identificação individual de qualquer natureza. Aos profissionais que concordaram participar foi solicitado que respondessem a um questionário sociodemográfico e a uma bateria de instrumentos psicométricos. Os questionários foram auto preenchidos em papel ou num formulário eletrónico disponível na página web do projeto, entre maio de 2011 e maio de 2013. A invariância de modo 'papel x internet' foi demonstrada para uma versão equivalente do MBI-HSS adaptada para estudantes universitários. ${ }^{16} \mathrm{O}$ estudo foi aprovado pelo Comité de Ética do Centro Hospital Lisboa Norte, EPE e pelo Comité de Ética da Unidade de Investigação em Psicologia e Saúde do ISPA-IU.

\section{Análise de Dados}

As qualidades psicométricas do MBI-HSS foram estudadas por recurso à análise fatorial confirmatória, e estatísticas derivadas, com o método de máxima verosimiIhança implementado no software AMOS (v. 21, SPSS An IBM Company, Chicago, IL). Considerou-se que o modelo de medida apresentou validade fatorial quando os índices usuais de qualidade de ajustamento do modelo foram $\chi^{2} / \mathrm{gl}$ $\leq 2$; $\mathrm{CFI} \geq 0,90$; GFI $\geq 0,90 ; \mathrm{TLI} \geq 0,90$; RMSEA $\leq 0,05 .{ }^{30} \mathrm{As}$ análises descritivas e inferenciais foram estratificadas por distrito/região autónoma recorrendo ao IBM SPSS Statistics (v. 21, SPSS An IBM Company, Chicago, IL). As comparações entre profissionais e distritos foram realizadas com uma ANOVA a 2-fatores depois de validado o pressuposto da homocedasticidade de variâncias. A associação entre o score de burnout e variáveis de contexto socioprofissional foi estimada com o coeficiente de correlação de Pearson (r) para variáveis quantitativas e o coeficiente de correlação de Spearman $\left(r_{s}\right)$ para variáveis ordinais. Utilizou-se um nível de significância de $5 \%$ para a tomada de decisão relativa à significância estatística dos resultados.

\section{RESULTADOS}

\section{Níveis de burnout em médicos e enfermeiros}

A distribuição percentual dos níveis burnout e o seu nível médio em médicos e enfermeiros, considerando as estimativas nacionais agregadas, encontram-se na Tabela 3.

Comparando os níveis de burnout em médicos e enfermeiros e a sua distribuição por distrito, verificaram-se diferenças estatisticamente significativas entre distritos ( $F$ $(15,1609)=2,04 ; p<0,05$, Potência $=0,968)$ existindo uma interação no limiar da significância entre a profissão e o distrito $(F(14,1609)=1,694 ; p=0,051$, Potência $=0,908)$. Não se observaram, porém, diferenças significativas entre os valores médios de burnout de médicos e enfermeiros $(F(1 ; 1609)=0,212 ; p=0,645$, Potência $=0,075)$ depois de considerar os efeitos do distrito e da interação entre distritos e profissão. A existência da interação significativa revela que os níveis médios de burnout por distrito diferem entre enfermeiros e médicos, recomendando a análise por distrito em cada grupo profissional separadamente.

A Fig. 1 apresenta os níveis médios de burnout em médicos e enfermeiros agrupados por classe de severidade de burnout (burnout reduzido, burnout moderado e burnout elevado) nos 20 distritos e regiões autónomas amostradas neste estudo.

Observou-se que em 10 (50\%) e 13 (65\%) dos 20 distritos/regiões autónomas os níveis de burnout foram elevados na classe dos médicos e enfermeiros, respetivamente. Os distritos do norte e do centro apresentam a maior concentração de burnout elevado em enfermeiros.

\section{Como explicar o burnout?}

A análise de algumas variáveis de contexto socioprofissional permite apontar alguns determinantes do burnout em profissionais da área da saúde. Em primeiro lugar obteve-se uma correlação estatisticamente significativa e negativa com o tempo na função quer em enfermeiros $(r=-0,142, p$ $=0,01)$ quer em médicos $(r=-0,151 ; p<0,001)$, observando-se que profissionais mais jovens (quer médicos quer enfermeiros) são mais acometidos por burnout (Fig. 2). Contudo, não se observou uma associação considerável entre o score de burnout e a duração da jornada de trabalho quer em enfermeiros $(r=0,06 ; p=0,03)$ quer em médicos

Tabela 3 - Média ( \pm desvio-padrão) e distribuição dos níveis de burnout de médicos e enfermeiros, a nível nacional que indicaram o distrito onde exerciam. Portugal, 2011-2013; $\mathrm{n}=1685$.

\begin{tabular}{lcc}
\hline Burnout & Médico(a) & Enfermeiro(a) \\
\hline Média \pm desvio-padrão & $2,9 \pm 1,67$ & $3,0 \pm 1,68$ \\
Burnout reduzido: score médio $<2(\%)$ & 32,4 & 29,9 \\
Burnout moderado: score médio [2;3[ (\%) & 24,0 & 20,8 \\
Burnout elevado: score médio $\geq 3(\%)$ & 43,6 & 49,4 \\
\hline
\end{tabular}


A

B

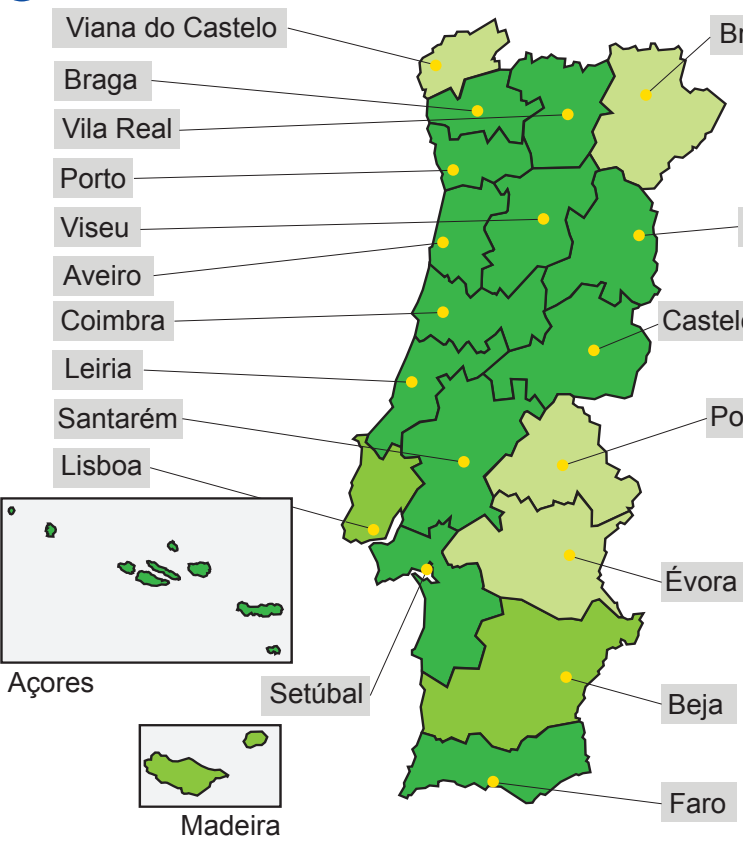

$<2$ (burnout reduzido)

[2; 3[ (burnout moderado)

$\geq 3$ (burnout elevado)

\section{Via}
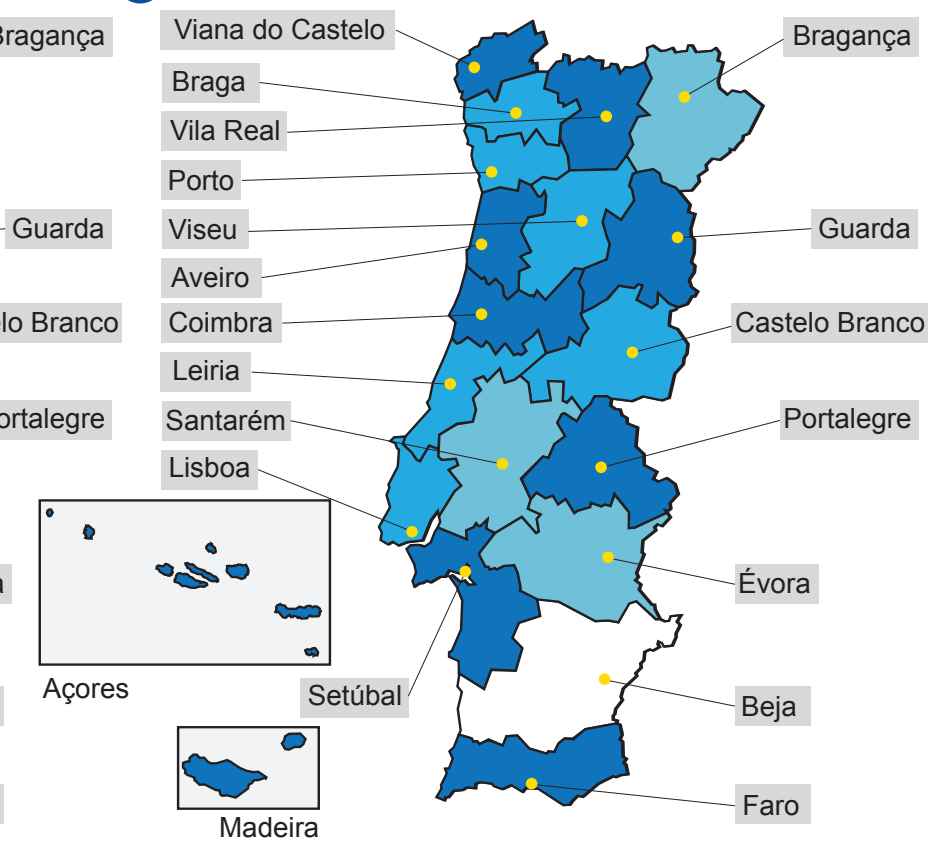

$<2$ (burnout reduzido)

[2; 3[ (burnout moderado)

$\geq 3$ (burnout elevado)

Figura 1 - Níveis médios de burnout por distrito/regiões autónomas em enfermeiros (A) e em médicos (B) (no Distrito de Beja não se obteve amostra suficiente para estimar com confiança o nível médio de burnout). Portugal, 2011-2013.

$(r=0,08 ; p>0,09)$.

Relativamente aos locais de trabalho, as diferenças observadas nos níveis de burnout não foram estatisticamente significativas quer em médicos, quer em enfermeiros ( $F(3$; $1622)=1,319 ; p=0,267$ ) (Fig. 3)

Entre as variáveis de contexto socioprofissional estudadas, aquela que revelou ser o melhor determinante de burnout foi a 'percepção das condições de trabalho'. Esta variável, avaliada numa escala de ' 1 - Muito más' a '5 Muito boas' revelou uma associação significativa e negativa moderada com os níveis de burnout quer em enfermeiros $\left(r_{\mathrm{s}}=-0,34 ; p=0,01\right)$ quer em médicos $\left(r_{\mathrm{s}}=-0,36 ; p=0,01\right)$ (Fig. 4).

Finalmente, no referente a diferenças entre géneros, não se observaram diferenças significativas nos níveis médios de burnout entre profissionais do género masculino ( $M$ $=2,99$, $\mathrm{DP}=1,69)$ e do género feminino $(\mathrm{M}=3,02$; $\mathrm{DP}=$ $1,67)(F(1 ; 1726)=0,063 ; p=0,802)$.

\section{DISCUSSÃO}

Este estudo apresenta dados relativos à incidência de burnout em médicos e enfermeiros com atuação em todos os distritos nacionais incluindo as regiões autónomas. Entre 2011 e 2013, os níveis de burnout em médicos e enfermeiros portugueses foram significativamente diferentes entre os distritos/regiões autónomas do país. O levantamento efetuado identificou um grande número de distritos com burnout elevado nas duas categorias profissionais ( $50 \%$ dos distritos para os médicos; $65 \%$ dos distritos para os enfermeiros). A similaridade estatística observada entre os níveis médios de burnout apresentado por médicos e enfermeiros portugueses está em linha com o estudo de

\section{Profissão}

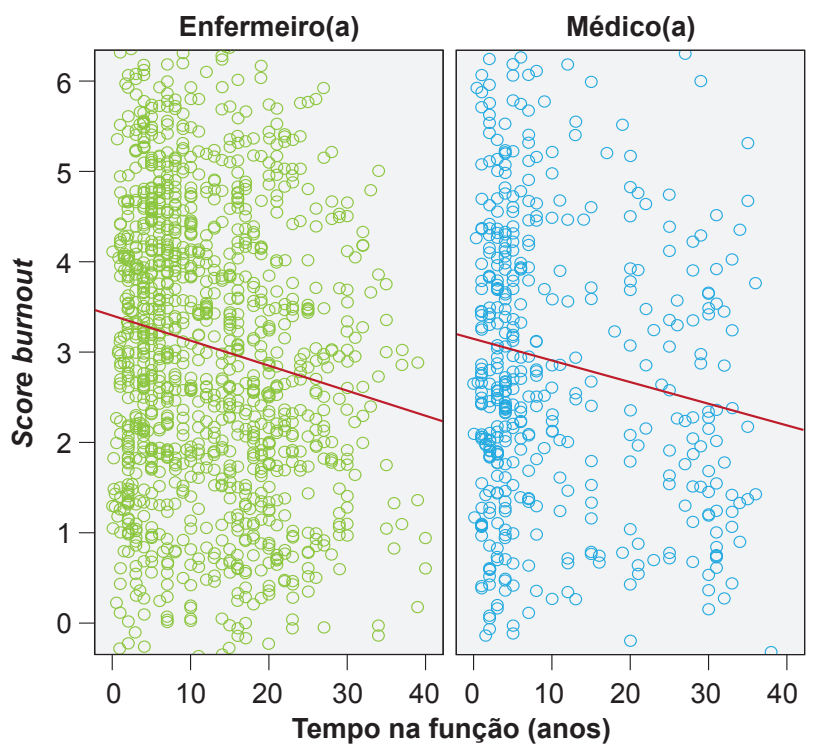

Figura 2 - Associação entre o score global de burnout e o tempo na função (anos) em enfermeiros e em médicos 
Profissão

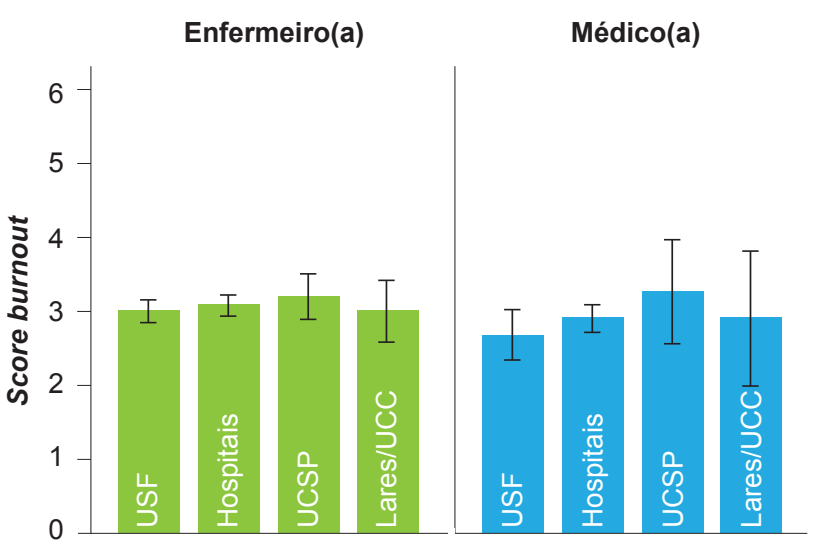

Local de trabalho

Figura 3 - Níveis médios ( \pm IC 95\%) de burnout por local de trabaIho em médicos e enfermeiros.

Tremolada et $\mathrm{al}^{26}$ que também referem a similaridade da atuação profissional destas duas profissões da área da saúde, frisando o alto risco de desenvolvimento de burnout em ambos os tipos de profissionais. O tempo de exercício na função e as condições de trabalho foram preditores significativos de burnout tanto em médicos quanto em enfermeiros. Os profissionais mais jovens apresentaram maiores níveis de burnout quando comparados com colegas com mais tempo de profissão. Estes resultados são consistentes com os resultados de Bilge ${ }^{31}$ que justifica esta observação com o facto de indivíduos com menor experiência profissional não terem ainda tempo suficiente para formular estratégias efetivas de coping para lidar com o stress ocupacional e por esse motivo estão mais vulneráveis ao desenvolvimento de burnout. Relativamente às condições de trabalho, Ferreira e Lucca ${ }^{4}$ corroboram os resultados apresentados neste estudo e os resultados de Bilge ${ }^{31}$ quando afirmam que a precariedade das condições de trabalho pode colocar os profissionais de saúde em risco relativamente à síndrome de burnout. Este facto pode ocorrer devido ao aumento do stress imposto para o exercício profissional em condições sub-ótimas para prestação do ato médico e ou de enfermagem..$^{32}$ Efetivamente, em Portugal, a perceção de más condições de trabalho foi o principal preditor da incidência de burnout nos profissionais de saúde avaliados.

Os resultados apresentados neste estudo pretendem alertar para a necessidade de mais investigação sobre o impacto das características laborais/organizacionais na área da saúde, nomeadamente sobre o bem-estar físico e psicológico dos profissionais de saúde. Mas, e também, para o possível compromisso da qualidade dos serviços prestados aos doentes. De facto, a investigação nesta área tem demostrado uma correlação negativa entre a dimensão de despersonalização/burnout de profissionais de saúde, a satisfação dos doentes e o tempo para a concessão de alta médica, depois de controlados os efeitos da severidade da condição do doente e os fatores demográficos. ${ }^{33}$

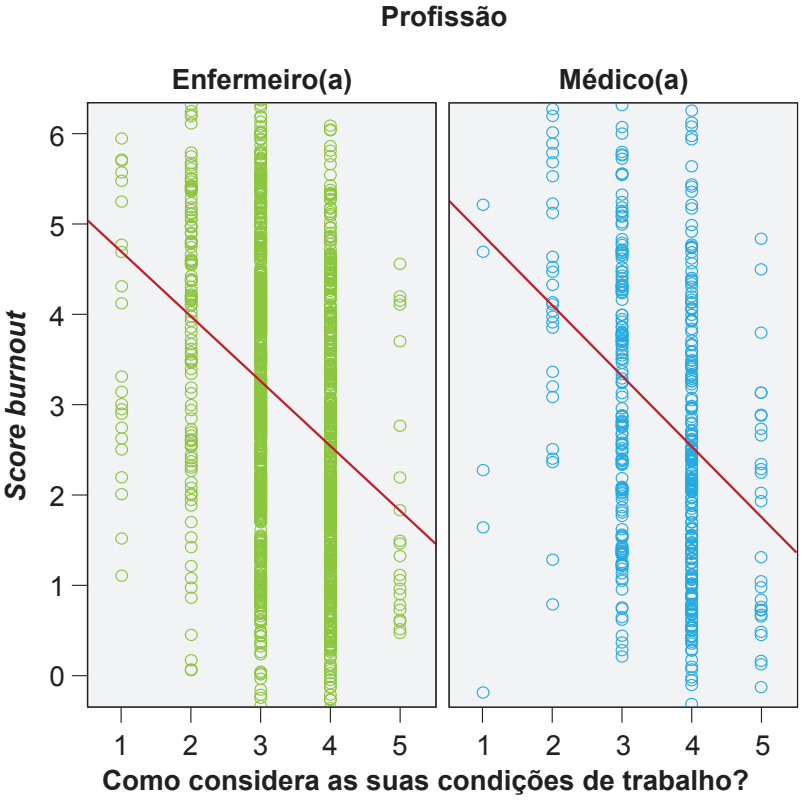

Figura 4 - Níveis médios de burnout em função da perceção das condições de trabalho (de '1 - Muito más' a '5 - Muito boas')

\section{CONCLUSÕES}

A nível nacional, entre 2011 e $2013,21,6 \%$ dos profissionais de saúde amostrados apresentaram burnout moderado e $47,8 \%$ burnout elevado.

Entre os médicos, a nível nacional, os níveis de burnout foram moderados, mas em 10 dos 20 distritos/regiões autónomas observou-se que os níveis médios de burnout foram elevados. Cerca de $44 \%$ dos médicos apresentaram burnout elevado. Os níveis médios de burnout em enfermeiros, em termos de média nacional, foram moderados. Porém em 13 dos 20 distritos/regiões autónomas os níveis de burnout dos enfermeiros foram elevados. Cerca de $50 \%$ dos enfermeiros amostrados apresentaram burnout elevado.

A perceção de más condições de trabalho foi, dos fatores estudados, o que melhor prediz a incidência de burnout tanto em médicos quanto em enfermeiros. Os resultados deste estudo, a nível nacional e com uma amostra de dimensão considerável, apontam para a necessidade de intervenções ocupacionais que reduzam a incidência do burnout em profissionais de saúde, melhorando o seu bem-estar físico e psicológico e potenciando a qualidade do serviço de saúde que estes prestam.

\section{AGRADECIMENTOS}

Ao ACES-Oeiras, Centro Hospitalar Lisboa Norte - EPE, à Ordem dos Enfermeiros, Associação dos Médicos de Família e Clinica Geral e ao Sindicato dos Médicos do Norte pela divulgação do estudo pelos seus associados.

\section{PROTECÇÃO DE PESSOAS E ANIMAIS}

Os autores declaram que os procedimentos seguidos estavam de acordo com os regulamentos estabelecidos pelo Comité de Ética do Centro Hospitalar Lisboa Norte, 
EPE e Comité de Ética da Unidade de Investigação em Psicologia e Saúde do ISPA-IU, em conformidade com a Declaração de Helsínquia da Associação Médica Mundial.

\section{CONFIDENCIALIDADE DOS DADOS}

Os autores declaram ter seguido os protocolos do seu centro de trabalho acerca da publicação de dados.

\section{REFERÊNCIAS}

1. Freudenberger HJ. Staff burn-out. J Soc Issues. 1974;30:159-65.

2. Maslach $\mathrm{C}$, Jackson SE. Maslach Burnout Inventory manual. Palo Alto: University of California, Consulting Psychologist Press; 1986.

3. Schaufeli WB, Leiter MP, Maslach C. Burnout: 35 years of research and practice. Career Dev Int. 2009;14:204-20.

4. Ferreira NN, Lucca SR. Síndrome de Burnout em técnicos de enfermagem de um hospital público do Estado de São Paulo. Rev Bras Epidemiol. 2015;18:68-79.

5. Wall M, Schenck-Gustafsson K, Minucci D, Sendén MG, Løvseth LT, Fridner A. Suicidal ideation among surgeons in Italy and Sweden - a cross-sectional study. BMC Psychol. 2014;2:53.

6. Shanafelt TD, Oreskovich MR, Dyrbye LN, Satele DV, Hanks JB, Sloan $\mathrm{JA}$, et al. Avoiding burnout: the personal health habits and wellness practices of US surgeons. Ann Surg. 2012;255:625-33.

7. Gil-Monte PR. Algunas razones para considerar los riesgos psicosociales en el trabajo y sus consecuencias en la salud pública. Rev Esp Salud Publica. 2009;83:169-73.

8. Rosenstein $\mathrm{AH}$. Physician stress and burnout: prevalence, cause, and effect. [consultado 2015 fev 25] Disponível em: http://www.aaos.org/ news/aaosnow/aug12/managing4.asp.

9. Goldberg R, Boss RW, Chan L, Goldberg J, Mallon WK, Moradzadeh $D$, et al. Burnout and its correlates in emergency physicians: four years' experience with a wellness booth. Acad Emerg Med. 1996;3:1156-64.

10. Shanafelt TD, Balch CM, Bechamps GJ, Russell T, Dyrbye L, Satele D, et al. Burnout and career satisfaction among American surgeons. Ann Surg. 2009;250:463-71.

11. Pinto AM, Chambel MJ. Burnout e engagement em contexto organizacional. Lisboa: Livros Horizonte; 2008.

12. Angelo RP, Chambel MJ. The reciprocal relationship between work characteristics and employee burnout and engagement: A longitudinal study of firefighters. Stress Health. 2015;31:105-14

13. Pedditzi ML, Nonnis M. Fonti psico-sociali di stress e burnout a scuola: una ricerca su un campione di docenti italiani. Med Lav. 2014;105:48-62.

14. Shinan-Altman $S$, Werner $P$, Cohen $M$. The connection between illness representations of Alzheimer's disease and burnout among social workers and nurses in nursing homes and hospitals: a mixed-methods investigation.Aging Ment Health. 2015:1-10.

15. Maroco J, Tecedeiro M. Inventário de burnout de Maslach para estudantes portugueses. Psicol Saúde Doenças. 2009;10:227-35.

16. Maroco J, Campos JA. Defining the student burnout construct: a structural analysis from three burnout inventories. Psychol Rep. 2012;111:814-30.

17. Gazelle G, Liebschutz JM, Riess H. Physician burnout: coaching a way out. J Gen Intern Med. 2015;30:508-13.

18. Lee HF, Yen M, Fetzer S, Chien TW. Predictors of burnout among nurses in Taiwan. Community Ment Health J. 2015;51:733-7.

\section{CONFLITOS DE INTERESSE}

Os autores declaram não terem qualquer conflito de interesse relativamente ao presente artigo.

\section{FONTES DE FINANCIAMENTO}

Os autores declaram não ter recebido subsídios ou bolsas para a elaboração do artigo.

19. Canadas-De la Fuente GA, Vargas C, San Luis C, Garcia I, Canadas $\mathrm{GR}$, De la Fuente El. Risk factors and prevalence of burnout syndrome in the nursing profession. Int J Nurs Stud. 2015;52:240-9.

20. Vargas C, Canadas GA, Aguayo R, Fernández R, De la Fuente EI. Which occupational risk factors are associated with burnout in nursing? A meta-analytic study. Int J Clin Health Psychol. 2014;14:28-38.

21. Howlett M, Doody K, Murray J, LeBlanc-Duchin D, Fraser J, Atkinson $P R$. Burnout in emergency department healthcare professionals is associated with coping style: a cross-sectional survey. Emerg Med J. 2015;32:722-7.

22. Khamisa N, Oldenburg B, Peltzer K, Ilic D. Work related stress, burnout, job satisfaction and general health of nurses. Int $\mathrm{J}$ Environ Res Public Health. 2015;12:652-66.

23. Kanai-Pak M, Aiken LH, Sloane DM, Poghosyan L. Poor work environments and nurse inexperience are associated with burnout, job dissatisfaction and quality deficits in Japanese hospitals. J Clin Nurs. 2008;17:3324-9.

24. Lorenz VR, Guirardello Ede B. The environment of professional practice and Burnout in nurses in primary healthcare. Rev Lat Am Enfermagem. 2014;22:926-33

25. Queiros C, Carlotto MS, Kaiseler M, Dias S, Pereira AM. Predictors of burnout among nurses: an interactionist approach. Psicothema 2013;25:330-5.

26. Tremolada M, Schiavo S, Tison T, Sormano E, De Silvestro G, Marson $\mathrm{P}$, et al. Stress, burnout, and job satisfaction in 470 health professionals in 98 apheresis units in Italy: a SIdEM collaborative study. J Clin Apher. 2015;30:297-304

27. Marcelino G, Cerveira JM, Carvalho I, Costa JA, Lopes M, Calado NE et al. Burnout levels among Portuguese family doctors: a nationwide survey. BMJ Open. 2012;2:pi:e001050.

28. Maslach C, Jackson SE, Leiter MP, Schaufeli WB, Schwab RL. Maslach Burnout Inventory Manual. Palo Alto: University of California, Consulting Psychologist Press; 1996.

29. Balogun JA, Helgemoe S, Pellegrini E, Hoeberlein T. Test-retest reliability of a psychometric instrument designed to measure physical therapy students' burnout. Percept Mot Skills. 1995;81:667-72.

30. Maroco J. Análise de equações estruturais: Fundamentos teóricos software \& aplicações. Pêro Pinheiro: Report Number; 2014.

31. Bilge F. Examining the burnout of academics in relation to job satisfaction and other factors. Soc Behav Pers. 2006;34:1151-60.

32. Bragard I, Dupuis G, Fleet R. Quality of work life, burnout, and stress in emergency department physicians: a qualitative review. Eur J Emerg Med. 2015;22:227-34

33. Halbesleben JR, Rathert C. Linking physician burnout and patien outcomes: exploring the dyadic relationship between physicians and patients. Health Care Manage Rev. 2008;33:29-39. 